Homology, Homotopy and Applications, vol.10(2), 2008, pp.1-12

\title{
REALIZATION THEOREMS FOR END OBSTRUCTIONS
}

\author{
BOGDAN VAJIAC
}

\author{
(communicated by Daniel Grayson)
}

\begin{abstract}
A stratified space is a filtered space with manifolds as its strata. Connolly and Vajiac proved an end theorem for stratified spaces, generalizing earlier results of Siebenmann and Quinn. Their main result states that there is a single $K$-theoretical obstruction to completing a tame-ended stratified space. A necessary condition to completeness is to find an exhaustion of the stratified space, i.e. an increasing sequence of stratified spaces with bicollared boundaries, whose union is the original space. In this paper we give an example of a stratified space that is not exhaustible. We also prove that the Connolly-Vajiac end obstructions can be realized.
\end{abstract}

\section{Introduction}

The results of this paper are closely related to the problem of characterizing those topological manifolds which can be the interior of a compact manifold with boundary. After the starting result due to Browder, Levine and Livesay in [5, L.C. Siebenmann showed that a topological manifold of dimension $n \geqslant 6, X$, which is tame at infinity, is the interior of a compact manifold with boundary if and only if a single obstruction $\sigma(X) \in \tilde{K}_{0}(\mathbb{Z} \pi)$ vanishes. Here $\pi$ denotes the fundamental group of the end of $X$.

An important contribution in this direction is due to F. Quinn ([29, 30, 32] $)$. He defines an obstruction, $q_{0}(X, A) \in \tilde{K}_{0}^{l f}\left(A, p_{X}\right)$, which vanishes if $A$ has a mapping cylinder neighborhood in $X$. Quinn's results generalize Siebenmann's theorem; in particular, a mapping cylinder neighborhood can be described as a "controlled" completion of $X-A$. We will review the definitions and the statement of Quinn's theorem in Section 3 below.

In 12 Connolly and Vajiac proved the following end theorem for stratified spaces:

Theorem. For any tame ended stratified space $X$ one can define a "controlled Quinntype" obstruction $\gamma_{*}(X)$. Assume it vanishes; i.e., $\gamma_{*}(X)=0$. Assume that the 5-dimensional skeleton, $X^{5}$, already has a completion; i.e., it is the interior of a compact stratified space. Then $X$ itself is the interior of a compact stratified space $\bar{X}$.

Received May 7, 2007, revised April 16, 2008; published on July 14, 2008.

2000 Mathematics Subject Classification: 57Q10; 57Q20, 57Q40, 57N40, 57N80.

Key words and phrases: stratified spaces, homology, homotopy.

This article is available at http://intlpress.com/HHA/v10/n2/a1

Copyright (C) 2008, International Press. Permission to copy for private use granted. 
The obstruction $\gamma_{*}(X)$ lies in a localization of the Quinn's group mentioned above, and it is a direct sum of obstructions (one for each stratum):

$$
\gamma_{*}(X)=\bigoplus_{m} \gamma_{m}(X) \in \underset{m}{\oplus} K_{0}^{l f}\left(\left(\hat{X}^{m-1}, p_{\hat{X}^{m}}\right)_{(\infty)}\right) .
$$

See 12 for more details on the definition of $\gamma$ and of the obstruction groups.

When a completion is impossible, one can ask at least to find an exhaustion of $X$. By this we mean an increasing sequence of compact stratified subspaces of $X$, with bicollared boundaries in $X$, whose union is $X$. Note that a stratified space that admits a completion is, in particular, exhaustible. Suppose now that $X$ is a stratified space with $n$-dimensional top stratum. Since the obstruction $\gamma_{*}(X)$ is a direct sum of obstructions, one for each stratum, we can concentrate our discussion on the top stratum of $X$. That is to say, assume that $X^{n-1}$, its $(n-1)$ dimensional skeleton, has a completion $Y$. Let $B=\partial Y$. Choose a collar neighborhood for $\partial Y$ in $Y$, say, $B \times(0, \infty] \subset Y$. Then the open cone of $B, O B$, can be identified to $B \times(0, \infty] / B \times\{\infty\}$. The open cone becomes a neighborhood of $\{\infty\}$ in $\hat{X}^{n-1}$, the one-point compactification of $X^{n-1}$. Let $\left.p_{\hat{X}}\right|_{O B}$ be the projection $\left.\operatorname{map} \operatorname{Holink}\left(\hat{X}, \hat{X}^{n-1}\right)\right|_{O B} \stackrel{\left.p_{\hat{X}}\right|_{O B}}{\longrightarrow} O B$ and denote by $p_{B}$ the restriction of $\left.p_{\hat{X}}\right|_{O B}$ to $B \times\{1\}$, where we identify $B$ with $B \times\{1\} \subset B \times(0, \infty) \subset X^{n-1}$. The Holink is discussed in detail in Section 2 .

In this particular case Theorem 1.12 in $[\mathbf{1 2}$ can be stated as follows:

Theorem (Exhaustibility Theorem from [12]). Let $X$ be a tame ended $n$-dimensional stratified space, $n \geqslant 6$, so that $X^{n-1}$ has a completion $Y$. Let $B=\partial Y$ be as above. Assume that $X^{5}$ admits an exhaustion and that all the fundamental groups of the holink fibers Holink $\left(X^{n}, X^{n-1}\right) \rightarrow X^{n-1}$ are good. Then there is an element $\partial \gamma_{n}(X) \in K_{-1}\left(B, p_{B}\right)$ so that $\partial \gamma_{n}(X)=0$ if and only if $X$ admits an exhaustion.

Remark 1.1. A group $G$ is $\operatorname{good}$ if $K_{i}(\mathbb{Z}[G])=0$ for $i \leqslant-2$. No example of a bad group is known.

The main results of this paper are Theorems 4.1 and 4.2. The first one provides a simple example of a stratified space which is not exhaustible.

Theorem (Theorem 4.1). Let $n \geqslant 3$. There exist manifolds $\widetilde{M}^{2 n+1}$, homeomorphic to $\mathbb{R}^{2 n+1}-\{0\}$, with a properly discontinuous cocompact semifree action of $C_{6} \times \mathbb{Z}$ so that:

(i) $\widetilde{M}^{C_{6}}$ is isomorphic to $\mathbb{R} \amalg \mathbb{R}$ and it is locally flat embedded in $\widetilde{M}$.

(ii) $\left(\widetilde{M}, C_{6}\right)$ has two ends; neither end is exhaustible (and therefore $\tilde{M}$ is not exhaustible).

(iii) $\left(\widetilde{M}, C_{6} \times \mathbb{Z}\right)$ is equivariantly $h$-cobordant to $\left(\mathbb{R}^{2 n+1}-\{0\}, C_{6} \times \mathbb{Z}\right)$. The action of $C_{6}$ is linear on $\mathbb{R}^{2 n+1}$, and $\mathbb{Z}$ acts (linear also) by multiplication by powers of two.

The second theorem states that any element in the group $K_{-1}\left(B, p_{B}\right)$ above can be realized in the form $\partial \gamma_{n}(W)$, for some stratified space $W$. Here is the precise statement: 
Theorem (Theorem 4.2). Let $Y$ be a compact n-dimensional stratified space. Denote by $\sigma Y$ its $(n-1)$-skeleton. Let $p_{Y}$ : Holink $(Y, \sigma Y) \rightarrow Y$ be the projection map. Denote by $\mathfrak{a}$ the forget-control assembly map ([35]):

$$
\tilde{K}_{-1}\left(\sigma Y, p_{Y}\right) \stackrel{\mathfrak{a}}{\longrightarrow} \tilde{K}_{-1}\left(\mathbb{Z} \pi_{1}(Y-\sigma Y)\right) .
$$

Let $x \in \operatorname{Ker}(\mathfrak{a})$ be an arbitrary element. Then there exists a stratified space $W$, containing $Y$, so that $\sigma W \approx \sigma Y \times \mathbb{R}$, and the obstruction to exhaust $W$ is $\partial \gamma_{n}(W)=$ $i_{*}(x)$. The map $i_{*}$ is induced by inclusion and gives an isomorphism between

$$
K_{-1}\left(\sigma Y, p_{Y}\right)=\tilde{K}_{0}^{l f}\left(\sigma Y \times \mathbb{R}, p_{Y} \times 1_{\mathbb{R}}\right) \quad \text { and } \quad \tilde{K}_{0}^{l f}\left(\sigma W, p_{W}\right) .
$$

The structure of this paper is the following: in Section 2 we review the theory of geometric modules and continuously controlled $K$-theory. More details are provided in 41 . Section 3 recalls the definition of the finiteness obstruction as introduced by F. Quinn in 30. We will use a more algebraic approach, as suggested by F. Quinn in 33 and Ranicki and Yamasaki in [35. We provide a quick account, for the reader's convenience. More details are provided in [41. We also discuss the relation between the torsion of an $h$-cobordism and the mapping cylinder obstructions.

The last section, Section 4, contains the proofs of the main theorems, Theorems 4.1 and 4.2 .

The author wants to thank his advisor, Professor Frank Connolly, for his guidance and support during the process of completing this work.

\section{Geometric modules and $K$-theory}

We will briefly recall the theory of geometric modules and the definition of the $K$-theory groups. These concepts were first introduced by Connell and Hollingsworth in 11. We follow an approach similar to the ones of Quinn ([29, 30. $)$ and Ranicki $(34,35)$. The full details are provided in 40 and $[41$.

Let $X$ be a topological space and let $\Pi(X)$ be the fundamental groupoid of $X$. This is a category with objects being the points of $X$ and morphisms from $x_{1}$ to $x_{0}$ being the homotopy classes of paths from $x_{0}$ to $x_{1}$.

The homotopy category of finite geometric modules on $X$ is:

$$
\mathcal{G}(X)=\operatorname{Ad} \mathbb{Z}[\Pi(X)]
$$

Here the categorical constructions $\operatorname{Ad} \mathbb{Z}[\Pi(X)]$ mean the abelian category generated by $\Pi(X)$. See 41 for a detailed description.

The continuously controlled $K$-theory of a pair $(X, B)$ was introduced in $\mathbf{2}$. Here is a very short explanation.

An abelian category over a space $X$, is an abelian category where every morphism is assigned a compact subset of $X$, called its support. The support of a morphism satisfies the following properties:

- $\operatorname{supp}(\sigma \circ \tau) \subset \operatorname{supp}(\sigma) \cup \operatorname{supp}(\tau)$.

- $\operatorname{supp}(\sigma+\tau) \subset \operatorname{supp}(\sigma) \cup \operatorname{supp}(\tau)$.

- If $\sigma=\sum_{i=1}^{k} n_{i} \sigma_{i}$, then $\operatorname{supp}(\sigma)$ is the union of the supports of those $\sigma_{i}$ for which $n_{i} \neq 0$. 
Definition 2.1. Let $B$ be a subspace of $X$. A collection, $\left\{S_{\lambda}: \lambda \in \Lambda\right\}$, of compact subsets of $X-B$, is continuously controlled, or $c c$ over $(X, B)$, if:

(a) For each $X$-neighborhood $U$ of $B,\left\{\lambda \in \Lambda: S_{\lambda} \not \subset U\right\}$ is a finite subset of $\Lambda$.

(b) For each point $p \in B$ and each $X$-neighborhood, $U(p)$, there is an $X$-neighborhood $V(p)$ so that any set $S_{\lambda}$ meeting $V$ must lie in $U$.

Using the continuously controlled notion, we define the homotopy category of geometric modules of a pair $\mathcal{G}(X, B)$ in a similar way. Objects in this category are pairs $(S, j)$, where $S$ is a set, and $j: S \rightarrow|\mathbb{C}|$ is a function for which the indexed collection $\{\operatorname{supp}(j(s)): s \in S\}$ is a $c c$-collection over $(X, B)$. For a morphism $f=\left(f_{t}^{s}\right)_{(s, t) \in S \times T}$, we require that $\left\{\operatorname{supp}\left(f_{t}^{s}\right):(s, t) \in S \times T\right\}$ is a $c c$-collection over $(X, B)$.

In particular, the locally finite geometric modules over $X$ are the geometric modules of the pair $(\hat{X}, \infty)$. Here $\hat{X}$ is a one point compactification of $X$.

Definition 2.2. Let $B$ be a subset of the topological space $X$. The groups $K_{i}(X, B)=K_{i}(\mathcal{G}(X, B)), i \leqslant 1$ are defined in the sense of Quillen (see [28] and [39]).

Similarly, $K_{i}^{l f}(X, B)=K_{i}(\mathcal{G}(\hat{X}, \hat{B})), K_{i}^{l f}(X)=K_{i}(\mathcal{G}(\hat{X}, \infty))=K_{i}\left(\mathcal{G}^{l f}(X)\right)$.

The reduced $K_{0}$-groups for these categories are defined as the cokernel of the natural map, $K_{0}(\mathcal{G}(X, B)) \rightarrow K_{0}\left(\mathcal{G}^{\text {id }}(X, B)\right)$, where the $\mathcal{G}^{\text {id }}(X, B)$ denotes the idempotent completion (see [34] for more details).

The Whitehead group, $\mathrm{Wh}(X, B)$ is defined as

$$
\mathrm{Wh}(X, B)=K_{1}(X, B) / H, \mathrm{Wh}(X)=\mathrm{Wh}(X, \emptyset)=K_{1}(\mathcal{G M}(X)) / H .
$$

Here $H$ is the subgroup of $K_{1}(X, B)$ generated by basis change matrices. Note that $\mathrm{Wh}(X) \approx \mathrm{Wh}\left(\pi_{1}\left(X, x_{0}\right)\right)$ if $X$ is path connected.

The Whitehead torsion of an isomorphism in $\mathcal{G}(X, B)$ is defined as usual:

$$
\tau(f)=[b \circ f] \in K_{1}(X, B) / H=\mathrm{Wh}(X, B) ;
$$

here $b$ is a base change isomorphism.

By using the ideas from [35] one can think of $\mathrm{Wh}(X, B)$ as the equivalence classes of contractible continuously controlled chain complexes over $\mathcal{G}(X, B)$. Hence an element in $\operatorname{Wh}(X, B)$ will be one of those complexes.

Since we will use the following notions, we recall a few definitions from [32], for the reader's convenience.

Definitions 2.3. Let $A$ be a subspace of a topological space $X$.

1. Holink.

$$
\operatorname{Holink}(X, A)=\left\{\sigma \in \operatorname{Map}([0,1], X) \mid \sigma^{-1}(A)=0\right\} .
$$

(It is a topological space, using the compact-open topology.) There are two evaluation maps:

$$
p_{X}: \operatorname{Holink}(X, A) \rightarrow A ; p_{X}(\sigma)=\sigma(0)
$$

and

$$
j_{X}: \operatorname{Holink}(X, A) \rightarrow(X-A) ; j_{X}(\sigma)=\sigma(1) .
$$


2. Forward tame. If $A$ is closed, then $A$ is said to be forward tame in $X$ if there exists a neighborhood $U$ of $A$ in $X$ a map $F: U \times[0, \infty] \rightarrow X$ such that $F^{-1}(A)=A \times[0, \infty] \cup U \times\{\infty\}$, and $F(a, t)=a, \forall(a, t) \in A \times[0, \infty]$.

3. Reverse tame. If $A$ is closed, then $A$ is said to be reverse tame in $X$ if there is a map $R:(X-A) \times[0, \infty] \rightarrow X-A$ so that:

(a) for each $t \in[0, \infty) \mathrm{Cl}_{X} R((X-A) \times[0, t]) \subset X-A$.

(b) each point $x \in X-A$ has a neighborhood $U$ and a number $t_{x} \in[0, \infty)$ so that $\left.R_{t}\right|_{U}=$ identity $\left.\right|_{U}$ for all $t \geqslant t_{x}$. Here $R_{t}: X-A \rightarrow X-A$ is defined by $R_{t}(x)=R(x, t)$.

(c) $R$ extends continuously to a map $(X-A) \times[0, \infty] \cup(A \times\{\infty\}) \rightarrow X$ by setting $R(a, \infty)=a, \forall a \in A$.

4. Tame. A closed subset of $X$ is tame if it is both forward and reverse tame.

5. Stratified space. A stratified space is a finitely filtered, locally compact Hausdorff space $\left(X,\left\{X_{i}\right\}_{0}^{n}\right)$ such that:

(a) Each stratum $X_{k}$ is a $k$-dimensional topological manifold (possibly with boundary).

(b) For each $i<j, X_{i}$ is tame in $X_{i} \cup X_{j}$.

(c) $\operatorname{Holink}\left(X_{i} \cup X_{j}, X_{i}\right) \stackrel{p}{\longrightarrow} X_{i}$ is a fibration, and the inclusion

$$
\left.\operatorname{Holink}\left(\partial X_{i} \cup \partial X_{j}, \partial X_{i}\right) \rightarrow \operatorname{Holink}\left(X_{i} \cup X_{j}, X_{i}\right)\right|_{\partial X_{i}}
$$

is a fiber homotopy equivalence over $\partial X_{i}$.

The $k$-th skeleton of a stratified space is defined as $X^{k}=X_{1} \cup X_{2} \cup \cdots \cup X_{k}$.

\section{Finiteness obstructions}

The controlled $\tilde{K}_{0}$-groups of the controlled finiteness obstruction were first introduced by Quinn in $[\mathbf{3 0}$. We will recall their definitions, using the algebraic approach of 33 and 35 . See also 40 and 41 . for more details.

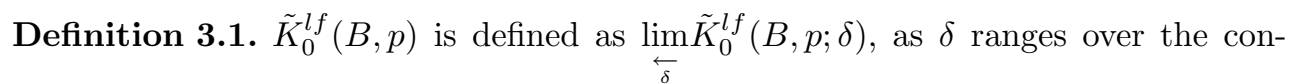
tinuous functions $\delta: B \rightarrow(0, \infty)$. (If $B$ is compact, then $\epsilon$ and $\delta$ can be chosen to be positive real numbers; in this case the $K_{0}$ groups will not have the locally finite superscript.)

The negative controlled $K$ groups of $(B, p)$ are defined ( $B$ compact) as

$$
\tilde{K}_{-i}(B, p)=\tilde{K}_{0}^{l f}\left(B \times \mathbb{R}^{i}, p \times 1_{\mathbb{R}^{i}}\right) .
$$

A few explanations are necessary about the above notation.

$p: E \rightarrow B$ is a continuous map from a topological space $E$ to a locally compact metric space $B$, and $\delta: B \rightarrow(0, \infty)$ is a continuous map. A subset $S \subset E$ is a $\delta$-subset if $p(S)$ lies in the $\delta(p(x))$ ball around $p(x)$, for each $x \in S$. A morphism is a $\delta$-morphism if $\operatorname{supp}\left(f_{t}^{s}\right)$ is a $\delta$-subset for each $(s, t)$. Following the ideas in the previous section, we can define the "category of $\delta$ geometric modules". A $\delta$-projective module is a pair $(M, e)$, where $e: M \rightarrow M$ has the property that $e^{2}$ is $\delta$-homotopic to $e$. The group $\tilde{K}_{0}^{l f}(B, p ; n, \delta)$ is defined as the set of equivalence classes of $\delta$-projective 
chain complexes of geometric modules. We suppress quite a few details. See $\mathbf{3 5}$, for a full account.

In the above notation, we will define now Quinn's mapping cylinder obstruction $([30)$. Let $C$ be an $n$-dimensional locally finite $\delta$-chain complex over $E$ which is $\delta$-dominated by a $\delta$-chain complex over $(B, p)$. In [35, Section 3.1], it is shown that $(C, 1)$ is $\epsilon$-chain equivalent to an $n$-dimensional $\epsilon$-projective chain complex, $(D, e)$, over $(B, p)$. The class

$$
q_{\delta}(C)=\chi([D, e]) \in K_{0}^{l f}(B, p ; 9 \delta)
$$

depends only on $C([\mathbf{3 5}, \mathbf{4 1}])$ and is called the $\delta$-controlled finiteness obstruction of $C$ over $B$.

Definition 3.2 (Quinn's mapping cylinder obstruction). Let $f: M \rightarrow B$ be a map from a manifold $M$ with compact boundary to a compact metric space $B$. Assume that $\operatorname{dim}(M) \geqslant 6$, and $(M, f)$ is tame ([29, Section 1.1]. Quinn defines the obstruction $q_{0}(M, f)$ as follows.

Let $M=M(0) \supset M(1) \supset M(2) \supset \cdots$ be an infinite sequence of submanifolds with compact boundaries and relatively compact complements in $M$. Assume that $\bigcap_{k=1}^{\infty} M(k)=\emptyset$. By using the tameness, one can choose this sequence so that $M(k)$ deforms, rel $\partial M(k)$, into the set $N(k)=M(k)-\operatorname{Int}(M(k+1))$, and the diameter of the deformations are $\langle\delta(k)$, where $\delta(1)>\delta(2)\rangle \cdots$, and $\lim _{k \rightarrow \infty} \delta(k)=0$. All measurements are made in $B$. Set $f(k)=f \mid M(k): M(k) \rightarrow B$.

Choose a handle decomposition of $(N(k), \partial M(k))$ and an infinite handle decomposition of $(M(k), \partial M(k))$ of diameter much less than $\delta(k)$. The chain complex $C(M(k), \partial M(k))$ is $\delta(k)$-finitely dominated by the finite complex $C(N(k), \partial M(k))$, so $q_{\epsilon(k)}(C(k))$ is defined as above. It turns out that the sequence

$$
q_{0}(M, f)=\left\{q_{\epsilon(k)}(C(k))\right\}_{k=1}^{\infty}
$$

is an element of the group: $\lim _{k} \tilde{K}_{0}(B, f(k), 9 \epsilon(k)) \approx \tilde{K}_{0}(B, p)$. Recall that $p$ is the holink projection. The element $q_{0}(M, f)$ is called the controlled end obstruction of $(M, f)$.

Remark 3.3. In [29, Chapter 7] and 30, Quinn proves that $q_{0}(M, f)=0$ if and only if $M$ is the interior of a compact manifold $\bar{M}$ such that $f$ extends to a map $\bar{f}: \bar{M} \rightarrow B$.

According to [1] (see also 41] for details), the following sequence

$$
\mathrm{Wh}(X, B) \stackrel{\Delta}{\longrightarrow} \tilde{K}_{0}(B, p) \stackrel{\mathfrak{a}}{\longrightarrow} \tilde{K}_{0}\left(\mathbb{Z} \pi_{1}(X-B)\right)
$$

is exact at $\tilde{K}_{0}(B, p)$.

A relative manifold is a compact Hausdorff pair $(X, A)$, for which $X-A$ is a paracompact manifold of dimension $\operatorname{dim}(X-A) \geqslant 5$, possibly with boundary, with $A$ tame in $X$.

A cobordism of relative manifolds between $(X, A)$ and $(Y, A)$ is a relative manifold $(W, A)$ such that $\partial W-A$ is the union of the two open sets $X-A$ and $Y-A$. 
An $h$-cobordism of relative manifolds is a cobordism $(W, A)$ between $(X, A)$ and $(Y, A)$ for which there are strict maps $r_{i}^{t}:(W, A) \rightarrow(W, A), 0 \leqslant t \leqslant 1, i=1,2$ deforming $(W, A)$ into $(X, A)$, respectively, $(Y, A)$, by a deformation which fixes $X$, respectively $Y$. The set of equivalence classes of $h$-cobordisms on $(X, A)$ is parametrized by $\mathrm{Wh}(X, A)($ 41] $)$.

We will denote Quinn's mapping cylinder obstruction of $A$ in $X$ by $q_{0}(X, A)$. (Recall that there is a map $X-A \rightarrow A$, given by the Holink.)

Theorem $3.4(41)$. Let $(X, B)$ be a relative manifold. Let $W$ be an $h$-cobordism of relative manifolds, from $(X, B)$ to $(Y, B)$. Let $\tau \in \mathrm{Wh}(X, B)$ be the torsion of $(W, B)$. Then

$$
\Delta(\tau)=r_{*} q_{0}(W, B)-q_{0}(X, B) .
$$

The map $\Delta$ is explicitly constructed in [41] and $r_{*}: \tilde{K}_{0}\left(B, p_{W}\right) \rightarrow K_{0}\left(B, p_{X}\right)$ is the isomorphism induced by the retraction $r$ from $W$ to $X$.

\section{A stratified space which is not exhaustible}

This section contains the two main results. They are closely related to Theorem 1.12 in [12], as was already mentioned in the introduction.

Recall that, for a stratified space $X$, an exhaustion means an increasing sequence of compact stratified subspaces of $X$, with bicollared boundaries in $X$, whose union is $X$.

Suppose now that $X^{n-1}$ has a completion $Y$. Let $B=\partial Y$. As explained in the introduction, Theorem 1.12 in $\left[\mathbf{1 2}\right.$ identifies an element $\partial \gamma_{n}(X) \in K_{-1}\left(B, p_{B}\right)$ so that $\partial \gamma_{n}(X)=0$ if and only if $X$ admits an exhaustion.

The goal of the present section is to describe a simple example of a tame ended stratified space which is not exhaustible. We then go on to prove that any element in the kernel of the assembly map can be represented, in a sense explained below, in the form $\partial \gamma_{n}\left(X^{\prime}\right)$, for some $X^{\prime}$. This can be viewed as a realization theorem for elements in $K_{-1}(B, p)$.

Let $C_{6}$ be the cyclic group of order 6 . Let $\mathbb{Z} C_{6}$ denote its group ring, equipped with the standard involution. It is known that $\tilde{K}_{0}\left(\mathbb{Z} C_{6}\right)=0$ and $K_{-1}\left(\mathbb{Z} C_{6}\right) \approx \mathbb{Z}$. Moreover, the standard involution acts trivially on $K_{-1}\left(\mathbb{Z} C_{6}\right)$. Hence every nonzero element $x \in K_{-1}\left(\mathbb{Z} C_{6}\right)$ satisfies the equation $x+\bar{x} \neq 0$, where $\bar{x}$ stands for the involution applied to $x$.

The proof of the fact that $\tilde{K}_{0}\left(\mathbb{Z} C_{6}\right)=0$ is number-theoretic in nature. It was first done (in a more general setting) by Bass and Murthy 4 . The method involves including $\mathbb{Z} C_{n}$ in the $\mathbb{Z}$-maximal order in $\mathbb{Q} C_{n}$ and carefully analyzing the kernel. See also 14 for a detailed exposition. The computation of $K_{-1}(\mathbb{Z} G)$, for $G$ finite abelian, is done in the book of H. Bass 3 . An easier approach that is specific to the group $C_{6}$ can be found in [36]. For complete details, refer to [3] or [36].

\section{Construction of the counterexample}

Theorem 4.1. Let $n \geqslant 3$. There exist manifolds $\widetilde{M}^{2 n+1}$, homeomorphic to $\mathbb{R}^{2 n+1}-\{0\}$, with a properly discontinuous cocompact semifree action of $C_{6} \times \mathbb{Z}$ so that: 
(i) $\widetilde{M}^{C_{6}}$ is isomorphic to $\mathbb{R} \amalg \mathbb{R}$ and it is locally flat embedded in $\widetilde{M}$.

(ii) $\left(\widetilde{M}, C_{6}\right)$ has two ends; neither end is exhaustible (and therefore $\tilde{M}$ is not exhaustible).

(iii) $\left(\widetilde{M}, C_{6} \times \mathbb{Z}\right)$ is equivariantly $h$-cobordant to $\left(\mathbb{R}^{2 n+1}-\{0\}, C_{6} \times \mathbb{Z}\right)$. The action of $C_{6}$ is linear on $\mathbb{R}^{2 n+1}$, and $\mathbb{Z}$ acts (linear also) by multiplication by powers of two.

Idea of proof. Let $V$ be a $(2 n+1)$-dimensional $\mathbb{R} C_{6}$ module so that $C_{6}$ acts semifreely, and $V^{C_{6}}$ is 1-dimensional. Let $\mathbb{Z}$ act on $V-\{0\}$ multiplying by powers of two. Set $M(V)=(V-\{0\}) / \mathbb{Z} . M(V)$ is a compact manifold which is (non-equivariantly) homeomorphic to $S^{1} \times S^{2 n}$. The $C_{6}$-fixed point set, $\sigma M(V)^{C_{6}}$, consists of two copies of $S^{1}$. The construction of $\widetilde{M}$ goes as follows: we will first alter $M(V)$ by an $h$-cobordism which will be a product cobordism on the singular set. At the other end of the $h$-cobordism we will get a $C_{6}$-manifold, say $M$, whose universal cover $\widetilde{M}$ will satisfy the conclusion of the theorem. The proof involves an analysis of the relationship between the Whitehead group parametrizing the $h$-cobordisms in question, and the mapping cylinder neighborhood obstruction of the singular set in the ambient manifold.

The proof relies on the following facts:

1. The $C_{6}$-equivariant $h$-cobordisms on $M(V)$, which are product cobordisms on $M(V)^{C_{6}}$, are parametrized by $\mathrm{Wh}\left(M(V) / C_{6}, M(V)^{C_{6}} / C_{6}\right)$. We will further denote the stratified space $M(V) / C_{6}$ by $X$ in order to simplify the notation. It follows that $\sigma X=X$ - top stratum $=M(V)^{C_{6}} / C_{6} \approx S^{1} \amalg S^{1}$.

2. There is an exact sequence

$$
\mathrm{Wh}(X, \sigma X) \stackrel{\partial}{\longrightarrow} \tilde{K}_{0}(\sigma X, p) \stackrel{j}{\longrightarrow} \tilde{K}_{0}\left(\mathbb{Z} \pi_{1}(X-\sigma X) .\right.
$$

See the previous section for references.

In our case it is easy to see that

$$
\tilde{K}_{0}\left(\mathbb{Z} \pi_{1}(X-\sigma X)\right) \approx \tilde{K}_{0}\left(C_{6} \times \mathbb{Z}\right) \approx \tilde{K}_{0}\left(\mathbb{Z} C_{6}\right) \oplus K_{-1}\left(\mathbb{Z} C_{6}\right) \approx \mathbb{Z} .
$$

This follows from the Bass-Heller-Swan formula and the previous observations. Also, $\tilde{K}_{0}\left(S^{1}, p\right) \approx \mathbb{Z}$. This can be accomplished either by an $E^{2}$-term computation, using Quinn's spectral sequence (see $\mathbf{3 0}$ ), or invoking the controlled version of the Bass-Heller-Swan formula (see [35, Chapter 7]). Note that, by the homogeneity of the above construction, the map

$$
p: \operatorname{Holink}(X, \sigma X) \rightarrow \sigma X
$$

is fiber homotopy equivalent to $p_{p t} \times 1_{S^{1}}$. It follows that the map $j$ above can be identified with

$$
\begin{gathered}
\mathbb{Z} \oplus \mathbb{Z} \stackrel{j}{\longrightarrow} \mathbb{Z}, \\
j(1,0)=j(0,1)=1 .
\end{gathered}
$$

3. The boundary map in the exact sequence above takes the torsion, $\tau$, of the homotopy equivalence between the two ends of an $h$-cobordism to the difference 
of the mapping cylinder obstructions of the singular sets for the two ends of the $h$-cobordism. More precisely: Let $W$ be a stratified $h$-cobordism from $X$ to $Y$, so that $\sigma W$ is homeomorphic to $\sigma X \times I$. Let $\tau=\tau(W, X) \in \mathrm{Wh}(X, \sigma X)$. Then

$$
\partial(\tau+\bar{\tau})=q_{0}(X, \sigma X)-r_{*} q_{0}(Y, \sigma Y) .
$$

This formula follows from Theorem 3.4

The proof is quite formal from now on.

Note that

$$
\operatorname{Ker}(j)=\{y \oplus(-y) \mid y \in \mathbb{Z}\} .
$$

Let $0 \neq x \in K_{-1}\left(\mathbb{Z} C_{6}\right)$. Then $(x \oplus(-x))=0$; hence there exist $\tau \in \mathrm{Wh}(X, \sigma X)$ so that $\partial \tau=x \oplus(-x)$. By the above $c c$-h-cobordism theorem, $\tau$ is the torsion of an $C_{6}$-equivariant $h$-cobordism from $\left(M(V), C_{6}\right)$ to a topological $C_{6}$-manifold, say $M$. By formula $(*)$ we have:

$$
q_{0}\left(M / C_{6}, M^{C_{6}} / C_{6}\right)=-\partial(\tau+\bar{\tau}) \neq 0 .
$$

One can easily conclude now that neither end of $\left(\widetilde{M}, C_{6}\right)$ has any stratified space neighborhoods. Recall that a stratified space neighborhood is a stratified space with bicollared boundary, required to be a neighborhood of only one end. Suppose that there exists a stratified space neighborhood $N$ of one of the ends of $\tilde{M}$, say $\varepsilon$. One can assume without loss of generality that

$$
(\sigma N, \sigma \partial N) \approx([0, \infty), 0)
$$

(If this is not the case, then one can pass to a smaller subneighborhood.) Consider the non-compact manifold $(\partial N-\sigma \partial N) / C_{6}$; because $\sigma \partial N$ is a point, it follows that the Siebenmann obstructions for the ends of this manifold, in $\tilde{K}_{0}\left(\mathbb{Z} C_{6}\right)$, are zero. So by [37], $\sigma \partial N$ has a mapping cylinder neighborhood $W$ in $\partial N$. By Quinn's collar extension theorem ([32 pp. 492-494]), the $C_{6}$-collar $[0, \infty)$ for $\sigma \partial N$ in $\sigma N$ extends to a $C_{6}$-collar of $\partial N$ in $N$. Similarly, if $N^{\prime}=\tilde{M}-\operatorname{Int}_{\tilde{M}} N, \partial N^{\prime}=\partial N$, then $\left(\sigma N^{\prime}, \sigma \partial N^{\prime}\right) \approx((-\infty, 0], 0)$. This is a collar for $\sigma \partial N^{\prime}$ in $\sigma N^{\prime}$, and it extends by the same argument to a collar for $\partial N^{\prime}$ in $N^{\prime}$. Therefore we have a neighborhood $U$ of $\sigma \tilde{M} \cup \partial N$ of the form $U=\mathbb{R} \times \partial N$ so that $\sigma U=\mathbb{R} \times \sigma \partial N . \mathbb{R} \times W$ is then a mapping cylinder neighborhood of $\sigma \tilde{M}$ in $\tilde{M}$. But this is absurd, by the above calculation.

\section{Realization theorem for elements of $K_{-1}\left(\sigma Y, p_{Y}\right)$}

Let $Y$ be a compact $n$-dimensional stratified space. Denote by $\sigma Y$ be its $(n-1)$ skeleton. Let $p_{Y}: \operatorname{Holink}(Y, \sigma Y) \rightarrow Y$ be the projection map. Denote by a the forgetcontrol assembly map (as defined above or see $\mathbf{3 5}$ ):

$$
\tilde{K}_{-1}\left(\sigma Y, p_{Y}\right) \stackrel{\mathfrak{a}}{\longrightarrow} \tilde{K}_{-1}\left(\mathbb{Z} \pi_{1}(Y-\sigma Y)\right) .
$$

Here is the main result:

Theorem 4.2. In the above notation, let $x \in \operatorname{Ker}(\mathfrak{a})$ be an arbitrary element. Then there exists a stratified space $W$, so that $\sigma W \approx \sigma Y \times \mathbb{R}$, and the obstruction to exhaust $W$, as explained before, is represented by $x$. This means that $\partial \gamma_{n}(X)=i_{*}(x)$. The 
map $i_{*}$ provides an (inclusion induced) isomorphism between $K_{-1}\left(\sigma Y, p_{Y}\right)$ and the group $\tilde{K}_{0}^{l f}\left(\sigma W, p_{W}\right)$. It will be explained below.

Proof. The argument is similar to the one used in the construction of the counterexample above. Here is the outline. First form $Y \times S^{1}$. It is clear that $\sigma Y \times S^{1}$ has a mapping cylinder neighborhood in $Y \times S^{1}$. This follows from an argument due to S. Ferry [17. See also Corollary 1.15 in $\mathbf{1 2}$. By [35, we have the isomorphism:

$$
\tilde{K}_{0}\left(\sigma Y \times S^{1}, p_{\left(Y \times S^{1}\right)}\right) \approx \tilde{K}_{0}\left(\sigma Y, p_{Y}\right) \oplus \tilde{K}_{-1}\left(\sigma Y, p_{Y}\right) .
$$

Look now at the exact sequence:

$$
\begin{aligned}
\mathrm{Wh}\left(Y \times S^{1}, \sigma Y \times S^{1}\right) & \stackrel{\Delta}{\longrightarrow} \tilde{K}_{0}\left(\sigma Y \times S^{1}, p_{Y \times S^{1}}\right) \\
& \stackrel{\mathfrak{a}}{\longrightarrow} \tilde{K}_{0}\left(\mathbb{Z} \pi_{1}\left(Y \times S^{1}-\left(\sigma Y \times S^{1}\right)\right)\right) \longrightarrow \cdots .
\end{aligned}
$$

Since $x \in \operatorname{Ker}(\mathfrak{a})$, it follows that there exists an element

$$
\tau \in \mathrm{Wh}\left(Y \times S^{1}, \sigma Y \times S^{1}\right),
$$

so that $\Delta(\tau)=x$. Construct an $h$-cobordism $W^{\prime}$ on $\left(Y \times S^{1}, \sigma Y \times S^{1}\right)$, so that, according to formula $(*)$ mentioned above, we have:

$$
q_{0}\left(W^{\prime}, \sigma W^{\prime}\right)=x .
$$

Take the infinite cyclic cover and get a stratified space $W$, with $\sigma W \approx \sigma Y \times \mathbb{R}$, so that the mapping cylinder obstruction of $\sigma W$ in $W$ is exactly as required. To be more precise, we should identify $K_{-1}\left(\sigma Y, p_{Y}\right)$ and $\tilde{K}_{0}^{l f}\left(\sigma W, p_{W}\right) \approx K_{-1}\left(\sigma Y,\left.p_{W}\right|_{\sigma Y}\right)$. But the construction provides a fiber homotopy equivalence between the corresponding holinks, and hence an induced isomorphism $i_{*}$ for the $K$-groups.

\section{References}

[1] D.R. Anderson, F.X. Connolly, and H.J. Munkholm, A comparison of continuously controlled and controlled $K$-theory, Topology and its Applications $\mathbf{7 1}$ (1996), no. 1, 9-46.

[2] D.R. Anderson, F.X. Connolly, S.C. Ferry, and E.K. Pedersen, Algebraic $K$-theory with continuous control at infinity, J. Pure Appl. Algebra 94 (1994), no. $1,25-47$.

[3] H. Bass, Algebraic K-theory, W. A. Benjamin, Inc., New York, 1968.

[4] H. Bass and M.P. Murthy, Grothendieck groups and Picard groups of abelian group rings, Ann. of Math. (2) 86 (1967) no. 1, 16-73.

[5] W. Browder, J. Levine, and G.R. Livesay, Finding a boundary for an open manifold, Amer. J. Math. 87 (1965), 1017-1028.

[6] D. W. Carter, Lower $K$-theory of finite groups, Comm. Algebra 8 (1980), no. 20, 1927-1937.

[7] T.A. Chapman, Topological invariance of Whitehead torsion, Amer. J. Math. 96 (1974), 488-497.

[8] T.A. Chapman, Homotopy conditions which detect simple homotopy equivalences, Pacific J. Math. 80 (1979), no. 1, 13-46. 
[9] T.A. Chapman, Controlled simple homotopy theory and applications, Lecture Notes in Mathematics 1009, Springer-Verlag, New York, 1983.

[10] M.M. Cohen, A course in simple-homotopy theory, Graduate Texts in Math. 10, Springer-Verlag, New York, 1973.

[11] E.H. Connell and J. Hollingsworth, Geometric groups and Whitehead torsion, Trans. Amer. Math. Soc. 140 (1969), 161-181.

[12] F. Connolly and B. Vajiac, An end theorem for stratified spaces, Invent. Math. 135 (1999), no. 3, 519-543.

[13] F. Connolly and B. Vajiac, A realization theorem for cylinder neighborhood obstructions, unpublished, 1996.

[14] C.W. Curtis and I. Reiner, Methods of representation theory. Vol. II. With applications to finite groups and orders, Pure and Applied Mathematics, A Wiley-Interscience Publication, John Wiley \& Sons, Inc., New York, 1987.

[15] F.T. Farrell and L.E. Jones, Isomorphism conjectures in algebraic $K$-theory, J. Amer. Math. Soc. 6 (1993), no. 2, 249-297.

[16] F.T. Farrell and L.E. Jones, The lower algebraic $K$-theory of virtually infinite cyclic groups, $K$-Theory 9 (1995), no. 1, 13-30.

[17] S. Ferry, A simple-homotopy approach to the finiteness obstruction, in Shape theory and geometric topology (Dubrovnik, 1981), pp. 73-81, Lecture Notes in Math. 870, Springer-Verlag, New York, 1981.

[18] S. Ferry, The homeomorphism group of a compact Hilbert cube manifold is an ANR, Ann. of Math. (2) 106 (1977), no. 1, 101-119.

[19] M.H. Freedman and F. Quinn, Topology of 4-manifolds, Princeton Mathematical Series 39, Princeton University Press, Princeton, NJ, 1990.

[20] B.C. Hughes, Geometric topology of stratified spaces, Electron. Res. Announc. Amer. Math. Soc. 2 (1996), no. 2, 73-81.

[21] M.A. Kervaire, Lectures on the theorem of Browder and Novikov and Siebenmann's thesis, Notes by K. Varadarajan. Tata Institute of Fundamental Research Lectures in Mathematics 46, Tata Institute of Fundamental Research, Bombay, 1969.

[22] R.C. Kirby and L.C. Siebenmann, Foundational essays on topological manifolds, smoothings, and triangulations, Annals of Mathematics Studies 88, Princeton University Press, Princeton, N.J., 1977.

[23] S. MacLane, Categories for the working mathematician, Graduate Texts in Mathematics 5, Springer-Verlag, New York, 1971.

[24] J. Milnor, Lectures on the h-cobordism theorem, Notes by L. Siebenmann and J. Sondow, Princeton University Press, Princeton, NJ, 1965.

[25] J. Milnor, Whitehead torsion, Bull. Amer. Math. Soc. 72 (1966), 358-426.

[26] E.K. Pedersen, On the bounded and thin $h$-cobordism theorem parameterized by $\mathbf{R}^{k}$, in Transformation groups (Poznań 1985), 306-320, Lecture Notes in Math. 1217, Springer-Verlag, New York, 1986. 
[27] E.K. Pedersen and C.A. Weibel, K-theory homology of spaces, in Algebraic topology (Arcata, CA, 1986), 346-361, Lecture Notes in Math. 1370, SpringerVerlag, New York, 1989.

[28] D. Quillen, Higher algebraic K-theory. I, in Higher K-theories (Proc. Conf. Battelle Memorial Inst., Seattle, Wash., 1972), Lecture Notes in Math. 341, 85-147, Springer-Verlag, New York, 1973.

[29] F. Quinn, Ends of maps. I, Ann. of Math. (2) 110 (1979), no. 2, 275-331.

[30] F. Quinn, Ends of maps. II, Invent. Math. 68 (1982), no. 3, 353-424.

[31] F. Quinn, Ends of maps. IV. Controlled pseudoisotopy, Amer. J. Math. 108 (1986), no. 5, 1139-1161.

[32] F. Quinn, Homotopically stratified sets, J. Amer. Math. Soc. 1 (1988), no. 2, 441-499.

[33] F. Quinn, Geometric algebra in Algebraic and geometric topology, Proceedings of the conference on surgery theory held at Rutgers University (New Brunswick, N.J., July 6-13, 1983) (A. Ranicki, N. Levitt and F. Quinn, eds.), Lecture Notes in Math. 1126, Springer-Verlag, New York, 1985

[34] A. Ranicki, The algebraic theory of finiteness obstruction, Math. Scand. 57 (1985), no. 1, 105-126.

[35] A. Ranicki and M. Yamasaki, Controlled K-theory, Topology Appl. 61 (1995), no. $1,1-59$.

[36] J. Rosenberg, Algebraic K-theory and its applications, Graduate Texts in Mathematics 147, Springer-Verlag, New York, 1994.

[37] L.C. Siebenmann, Obstructions to finding a boundary for an open manifold, Ph.D. Thesis, Princeton University, 1965.

[38] L.C. Siebenmann, Infinite simple homotopy types, Nederl. Akad. Wetensch. Proc. Ser. A 73, Indag. Math. 32 (1970), 479-495.

[39] V. Srinivas, Algebraic K-theory, Second edition, Progress in Mathematics 90, Birkhuser Boston, Inc. Boston, MA, 1996.

[40] B. Vajiac, University of Notre Dame Ph.D. Thesis, 1997.

[41] B. Vajiac, $H$-cobordisms and mapping cylinder obstructions, Journal of the Australian Mathematical Society, to appear.

[42] C.T.C. Wall, Surgery on compact manifolds, London Math. Soc. Monographs, No. 1, Academic Press, New York, 1970.

[43] S. Weinberger, The topological classification of stratified spaces, Chicago Lectures in Mathematics, Chicago University Press, 1994.

Bogdan Vajiac bvajiac@iun.edu

Department of Mathematics, Saint Mary's College, Notre Dame, Indiana 46556, USA 